\title{
PAPER \\ Robust Iris Segmentation Based on Local Image Gradient Properties
}

\author{
Somying THAINIMIT ${ }^{\dagger a}$, Member, Chirayuth SREECHOLPECH $^{\dagger}$, Vuttipong AREEKUL ${ }^{\dagger}$, \\ and Chee-Hung Henry $\mathrm{CHU}^{\dagger \dagger}$, Nonmembers
}

\begin{abstract}
SUMMARY Iris recognition is an important biometric method for personal identification. The accuracy of an iris recognition system highly depends on the success of an iris segmentation step. In this paper, a robust and accurate iris segmentation algorithm for closed-up NIR eye images is developed. The proposed method addressed problems of different characteristics of iris databases using local image properties. A precise pupil boundary is located with an adaptive thresholding combined with a gradient-based refinement approach. A new criteria, called a local signal-to-noise ratio (LSNR) of an edge map of an eye image is proposed for localization of the iris's outer boundary. The boundary is modeled with a weighted circular integral of LSNR optimization technique. The proposed method is experimented with multiple iris databases. The obtained results demonstrated that the proposed iris segmentation method is robust and desirable. The proposed method accurately segments iris region, excluding eyelids, eyelashes and light reflections against multiple iris databases without parameter tunings. The proposed iris segmentation method reduced false negative rate of the iris recognition system by half, compared to results obtained using Masek's method.

key words: iris localization, iris segmentation, NIR iris images, local signal-to-noise ratio
\end{abstract}

\section{Introduction}

Automatic, secure personal identification and authentication system based on biometrics have seen more widespread adoptions recently. Among the biometrics available, the iris recognition system yields a very high level of accurate identification. Iris recognition uses iris patterns, which are formed during the eighth month of gestation, to achieve identification of individuals. The formation is believed to generate highly unique, distinguishable and reliable iris patterns [1], suitable for personal authentication. The human iris is a thin, colored, contractile and circular disc, suspended between the cornea and the lens [2] as shown in Fig. 1. These eye images are examples of typical input images of an iris recognition system. Generally, iris acquisition systems acquire images with the assistance of near-infrared (NIR) light source(s). Under the NIR condition, texture details of irises can be seen clearer.

A typical iris recognition system is comprised of four main modules: acquisition, preprocessing, feature extraction, and feature matching. During the acquisition phase, a

Manuscript received June 29, 2010.

Manuscript revised September 22, 2010.

${ }^{\dagger}$ The authors are with Electrical Engineering Dept., Faculty of Engineering, Kasetsart Univ., Bangkok, 10900 Thailand.

${ }^{\dagger}$ The author is with University of Louisiana at Lafayette, Lafayette, LA 70504-4330, USA.

a)E-mail: fengsyt@ku.ac.th

DOI: 10.1587/transinf.E94.D.349

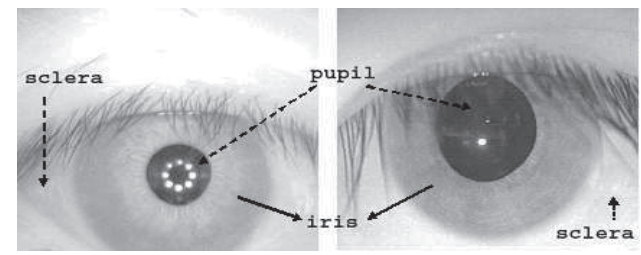

Fig. 1 Examples of NIR eye images (a) The CASIA-irisv.3-interval database, (b) The KSIP-db01R database.

sequence of eye images of a subject is captured. These images are preprocessed with three main steps: segmentation, normalization, and enhancement. The purpose of segmentation is to isolate an iris region of the eye from the image. Generally, this is done by detecting and modeling an inner (pupil) and outer (sclera) iris boundaries. This detection process is often called iris localization. After localization, irrelevant portions such as eyelids, eyelashes and reflections are removed. Then, the segmented iris region are normalized and enhanced to provide an effective region for subsequent processes. In the feature extraction module, the goal is to generate an iris code that represents the identity of each individual. A person's identity is determined by the similarity of the generated template and the reference iris codes stored in a database.

The success of the segmentation module is crucial to the performance of an iris recognition system, since incorrectly segmented regions can mislead subsequent processes. Factors that affect the segmentation accuracy include the quality of captured eye images such as the eye's size, occlusions, and its focus quality. Typically, different iris databases have different image qualities such as its ambient illumination, iris's size, eyelid/eyelash obscuration, etc. This variability poses more challenges in the development of an iris segmentation algorithm that are robust across the divergent iris databases.

In the literature, iris segmentation methods have been classified into two categories: a model-based method and an active contour-based method. The model-based method approximates iris boundaries using a parametric shape such as a circle or an ellipse, whereas the active contour-based method approximates the iris boundaries with closed contours. The active contour-based approach is more suitable for iris images with severe occlusions and distorted in shape. However, the shape of the obtained iris boundaries will not be elliptical or circular. Hence, the obtained boundaries will 
complicate the subsequent iris recognition process. Additionally, the International Organization for Standardization have recommended a circular model for a polar iris image specification [3].

In the best known iris recognition system presented by Daugman [1], both iris boundaries are localized by the optimization of the integro-differential equation:

$$
\max _{\left(r, x_{0}, y_{0}\right)}\left|G_{\sigma}(r) * \frac{\partial}{\partial r} \oint_{\left(r, x_{0}, y_{0}\right)} \frac{I(x, y)}{2 \pi r} d s\right|
$$

where $G(r)$ represents a Guassian operator of scale $\sigma, I$ is an input eye image, and a contour integral along a candidate circle given by radius $r$ and center $\left(x_{0}, y_{0}\right)$. A later paper by Daugman [4] elaborates on the development of an active contour segmentation method based on the discrete Fourier series expansion. The active contour approach avoids any assumptions on the parametric shape of an iris. Other active contour-based approaches have been developed: Ross [5] used the evolution of the geodesic active contours to dynamically fit the iris boundaries; Arvacheh [6] used a snake model to obtain a near-circular shape of the iris boundary; He et al. [7] used an elastic model called pulling and pushing to locate non-circular iris boundaries. Tan et al. [8] proposed an integro-differential constellation approach to accelerate the conventional equation aforementioned. Circular iris boundaries are then refined with the application of intensity statistics. Sankowski et al. [9] also exploited the conventional integro-differential for iris localization. He proposed to utilize information in different image color spaces. The iris outer boundary is located in the Y component, whereas the iris inner boundary is located in the red component of the eye image.

Another well-known iris localization method is proposed by Wild [10]. His approach is a gradient based approach combined with a parametric Hough transform. An edge map of an iris image is first obtained by thresholding the magnitude of the first derivative of the image, followed by the Hough transform. Several subsequent works [11]-[18] follow Wild's approach with some variations: Liu et al. [11] improved the accuracy of iris localization by the prior reduction of irrelevant edge points of the iris boundaries. Proenca and Alexandre [12] proposed to produce a more robust edge map from clustered pixels of an iris image. The clustered image is produced with momentbased texture segmentation and K-means clustering algorithms. L. Masek [13] implemented an open source of an iris recognition algorithm. A canny edge detector is used to obtain an edge map of the eye image. The iris boundaries are modeled with the circular Hough transform. The eyelids are localized by the linear Hough transform. A conventional thresholding operator is used for the eyelashes removal.

One major problem of most existent edge-based approaches is on accurately detecting the sclera boundary, since this boundary has a soft contrast in the NIR-iris images. An application of global edge detection always results in incomplete edges around the sclera boundary with strong edges of eyelids, eyelashes and pupil. These strong edges cause interference on the segmentation process, resultant in an inaccurate sclera boundary.

This paper focuses on the development of an edgebased iris segmentation scheme that works efficiently on multiple NIR-iris image databases. The iris boundaries are modeled using two non-concentric circles. The model is chosen in adherence to the ISO M1 Iris image data standards [3]. The proposed segmentation method exploits local properties of an image for iris segmentation. Threshold value used in pupil detection is automatically adapted to the minimum value of local means of eye sub-images. A reliable sclera boundary is accomplished via a local signal-tonoise ratio (LSNR) of an edge map of an input eye image. The proposed local properties are able to handle inconsistencies present in diverse iris databases. Despite the absence of any conventionally necessary parameter tunings, the proposed method achieves high accuracy results with two distinct iris databases: CASIA-irisv.3-interval [19] and KSIP-db01R [20]. Examples of eye images used in our experiments are shown in Fig. 1. Major differences among the two iris databases are illumination backgrounds and occlusion conditions.CASIA3.0 employs higher levels of ambient illumination as well as more NIR-LEDs than KSIP. This leads to difficulties in accurate pupil localization as the pupil boundary is disturbed by the LED light. Images in KSIP suffer of higher occlusions than images of CASIA3.0. The edges of eyelashes often degrade the performance of the iris boundary localization.

\section{Iris Localization Using Local Image Properties}

Most present edge-based iris localization approaches exploit global features, for example global thresholding of image intensity or its edge map. These methods assume a small variability of illumination conditions of the iris images. However, these conditions are often invalid for multiple iris databases. Dissimilar iris databases generally captured eye images under different conditions. Therefore, parameters of the segmentation system applied with each database must be determined prior to its usage. To overcome this problem, local features that are less sensitive to the inconsistency of iris's illumination conditions are proposed for robust iris segmentation of multiple iris databases. Details of the proposed method are elaborated in following sections.

\subsection{Pupil Boundary Localization}

Typically, an NIR eye image has a bimodal histogram in which the lower part of the histogram mainly associates with the pixels of a pupil, eyelashes and eyebrows. Therefore, a thresholding technique is applicable to pupil segmentation. However, global thresholding is sensitive to illumination of an image. Therefore, an appropriate threshold value based on its illumination background and interferences of each iris database must be determined prior the application. To avoid this training process, we propose a self adaptive thresholding based on the minimum value of local mean values for 


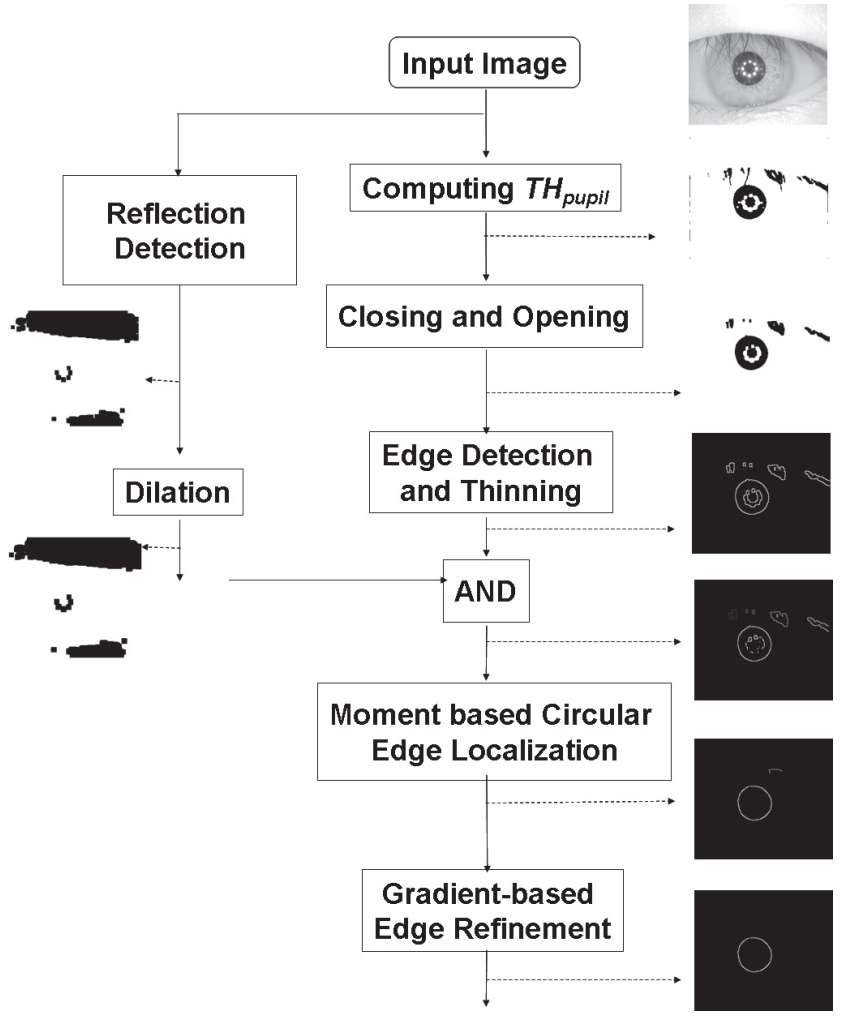

Fig. 2 The flowchart of the proposed pupil localization with its corresponding results.

coarse pupil detection, combined with a gradient-based refinement procedure to improve the segmentation accuracy.

Figure 2 illustrates the proposed pupil localization scheme. The images on the left- and right-hand side depict the obtained result of each step. The method starts by the division of an iris image into sub-images sized $16 \times 16$ pixels. Subsequently, an average intensity of each sub-image is calculated. The pupil region is segmented with the minimum value among the obtained means. The segmented threshold value $\left(T H_{\text {pupil }}\right)$ is formulated as:

$$
T H_{\text {pupil }}=\operatorname{argmin}_{R \subseteq I} E(R)
$$

where $R$ ranges over all sub-windows in image $I$ and $E(R)$ is the average value of the region $R$. Since the minimum value is used, the pupil region is always contained in the segmented result. However, the obtained region is often segregated into upper and lower parts. Morphology closing is used to merge the two parts. Then morphology opening is applied to isolate eyelashes and other irrelevant noises from the detected pupil region.

To determine the pupil boundary, an edge operator (such as the Sobel edge detector) along with a process of thinning is applied. The obtained edges often include edges of the pupil boundary and other irrelevant interferences such as edges of light reflections and eyelashes. Edges of light reflections are removed by the deletion of edges associated with high intensity values, since those values are often caused by light reflections. Pixels associated to the light reflections are detected using thresholding, followed by dilation operation. The dilated result is then combined with the result obtained from the thinning operation using AND operator. Other irrelevant edges are further removed based on the circularity property of the pupil. A centroid of the remaining edges is computed and serves as the approximate center of the detected circle. Next, edges with improper circular direction based on the center obtained are removed. As NIR-iris images provide high contrast pupil boundaries, more accurate pupil boundaries are obtained by the search for edges with the maximum gradient values within a prespecified search area. Finally, the detected edges are modeled with a circular model fitting technique [21]. The average computational time of the pupil boundary localization on a personal computer (Core2Duo $2.20 \mathrm{GHz}$ with $1 \mathrm{~GB}$ RAM) is 0.325 seconds.

\subsection{The Sclera Boundary Localization}

The tendency of soft contrast of the sclera boundary of an NIR image often causes a problem for the iris outer boundary localization. Typical edge detectors used in edgebase iris localization techniques often result in partial edges. Even when an enhancement operator is employed to improve the contrast of the boundary, it also generates unwanted spurious edges which confuse the modeling process.

\subsubsection{The Sclera Boundary Detection}

To address the aforementioned problem, an enhancement of soft contrast sclera boundary with a local signal-to-noise ratio (LSNR) of the edge map of an eye image is proposed. In this paper, the edge map image is computed using the Sobel edge operator. The LSNR is a ratio of a local mean to a local standard deviation. From pervious experiments, the LSNR of an edge map is very robust to illuminate the variations among iris databases. The LSNR technique can reliably detect an iris outer boundary.

For an image $I$, its local area is defined as a $(2 m+1) \times$ $(2 n+1)$ window centered at pixel $(x, y)$, where $m, n$ is an integer number. The local mean and local standard deviation of pixel $(x, y)$ can be computed with Eq. (3) and Eq. (4), respectively.

$$
L M(x, y)=\frac{1}{(2 m+1)(2 n+1)} \sum_{j=-m}^{m} \sum_{i=-n}^{n} I(x+i, y+j)
$$

$$
\begin{aligned}
& \operatorname{LS} D(x, y)= \\
& \qquad \sqrt{\frac{1}{(2 m+1)(2 n+1)} \sum_{j=-m}^{m} \sum_{i=-n}^{n}[I(x+i, y+j)-L M(x, y)]^{2}}
\end{aligned}
$$

The LSNR of a pixel $(\mathrm{x}, \mathrm{y})$ is then computed using Eq. (5). 




(a)



(c)

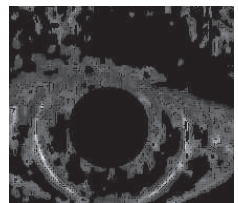

(e)



(b)



(d)



(f)
Fig. 3 (a) local mean of an edge image, (b) local standard deviation of the edge image, (c) a LSNR edge image, (d) result after the removal of pixels of a very low mean and standard deviation, (e) result after the removal of pixels associated with the pupil region, (f) result after the removal of pixels of improper circular direction.

$$
\operatorname{LSNR}(x, y)=20 \log \frac{\operatorname{LM}(x, y)}{\operatorname{LS} D(x, y)} d B
$$

To accelerate the system computational time, the $\operatorname{LSNR}(x, y)$ applied is reduced to Eq. (6).

$$
\operatorname{LSNR}(x, y)=\frac{\operatorname{LM}(x, y)}{\operatorname{LS} D(x, y)}
$$

To obtain the LSNR values of an edge map, the average and standard deviation values of the obtained edge map are locally computed with a $11 \times 11$ pixel window. The size of window is obtained empirically. Figure 3 (a) and 3 (b) depict example results of the obtained local mean and local standard deviation of an edge map. It is evident that edges of pupil display high local means and high local standard deviations. Edges of the sclera have high local means but low local standard deviations due to their lower contrast levels.

Motivated by these observations, the LSNR of the obtained edge map is computed using Eq. (6). The division of the local average value with its relevant local standard deviation enhances edges of sclera boundary significantly as shown in Fig. 3 (c). To avoid zero division, edge pixels with very low local means and standard deviations are eliminated prior the division. The obtained result, as shown in Fig. 3 (d), illustrates that these edges often belong to smooth regions of the eye image. Next, pixels related to the pupil region obtained from the previous section are removed, which yields the result shown in Fig. 3 (e). Lastly, the directions of the obtained edges are investigated. Edges with improper circular direction associated to the directions of edges of the pupil are removed. The obtained LSNR of the edge map is shown in Fig. 3 (f).

\begin{tabular}{|l|l|l|}
\hline 1 & -2 & 1 \\
\hline 1 & -2 & 1 \\
\hline 1 & -2 & 1 \\
\hline
\end{tabular}

(a)

\begin{tabular}{|l|l|l|}
\hline 1 & 1 & 1 \\
\hline-2 & -2 & -2 \\
\hline 1 & 1 & 1 \\
\hline
\end{tabular}

(b)
Fig. 4 (a) Vertical dark line detector (b) Horizontal dark line detector.

\subsubsection{The Sclera Boundary Modeling}

To model an outer iris boundary, a weighted circular integral of the attained LSNR values of an edge map is exploited. The modeling is performed by the optimization of the below equation:

$$
\max _{\left(r, x_{0}, y_{0}\right)} \oint_{\left(r, x_{0}, y_{0}\right)} w_{x_{0}, y_{0}} \frac{\operatorname{LSNR} R_{\text {edge }}(x, y)}{2 \pi r} d s
$$

where $L S N R_{\text {edge }}$ is a LSNR of an edge map of an input eye image, and $w$ is a weight value used to constrain a center of the outer boundary of the iris. The center of the outer boundary should be as close as feasible to the center of the pupil. Thus, the weight value is defined by following equation:

$$
w_{i}=1-\frac{d_{i}}{d_{\max }}
$$

where $d_{i}$ is the distance between the center of the pupil and the center of candidate circle $i$, and $d_{\max }$ is the allowable tolerance between the center of the pupil and the center of possible circles in the search area. This optimization renders the maximum contour integral over a summation of the $L S N R$ along a candidate circle given by $r$ radius and center $\left(x_{0}, y_{0}\right)$ over the obtained LSNR edge image. The proposed sclera boundary localization consumes more times than the pupil boundary localization. The average computational time of the process is 0.929 seconds.

\subsubsection{Eyelash and Eyelid Removal}

Once the iris boundaries are localized, major occlusions of an iris, for instance eyelashes and eyelids, are removed. An adaptive thresholding is used in this paper to detect separable and multiple eyelashes. A separable eyelash is a single eyelash of a thin line shape. Multiple eyelashes are a group of separable eyelashes which are connected. For separable eyelashes, a vertical dark line detector mask, as shown in Fig. 4 (a) is applied over an eye image. Its average and standard deviation values are computed. The separable eyelashes are detected by thresholding the obtained image with the threshold value of the computed mean in addition to three times of its standard deviation. Then, multiple eyelashes are located by thresholding the image based on the average and standard deviation values of the detected separable eyelashes. The threshold value is derived from the obtained average value minus its standard deviation. 




(a)

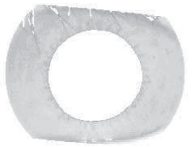

(b)
Fig. 5 Examples of the segmented irises.

For the detection of eyelids, a horizontal dark line detector, as illustrated in Fig. 4 (b), is applied over an eye image. This detector is suitable for the eyelid detection since the boundary between the iris and the eyelids is usually darker than their pixels. The result acquired from the detection, denoted by $G(x, y)$ is consequentially modeled with a weight parabolic integral defined as follow:

$$
\max _{\left(p, \theta, x_{v}, y_{v}\right)} \oint_{\left(p, \theta, x_{v}, y_{v}\right)} w_{x_{v}} \frac{G(x, y)}{l} d s
$$

This approach is analogous to Eq. (7), except that the integral is performed along the parabolic curve characterized by parameters $p, \theta, x_{v}$, and $y_{v}$, where $\left(x_{v}, y_{v}\right)$ indicates the vertex of a parabola, $p$ indicates the distance between the vertex and the focus of the parabola, and $\theta$ indicates the angle of the parabola. An equation of the parabolic curve is defined by Eq. (10). The $l$ is a latus rectum of the parabola. The weight value is computed with Eq. (8), but in this instance $d_{i}$ is the difference between the row index of the iris center and the row index of the parabola vertex, and $d_{\text {max }}$ is the maximum allowable $d_{i}$ in the search space.

$$
\begin{aligned}
& \left(\left(y-y_{v}\right) \cos \theta-\left(x-x_{v}\right) \sin \theta\right)^{2}= \\
& \quad 4 p\left(\left(y-y_{v}\right) \sin \theta+\left(x-x_{v}\right) \cos \theta\right)
\end{aligned}
$$

Figure 5 displays examples of the attained segmentation images.

\section{Performance Evaluations}

Three experiments were set up to evaluate the proposed iris segmentation approach. The first two experiments measured the accuracy of the suggested localization approach. The first experiment served to visually inspection accuracy of the approach. The second experiment validate system accuracy with the application of classification errors criteria. The third experiment was designed to validate the effects of the proposed segmentation approach on the accuracy of iris recognition systems. In all experiments, the results of the proposed segmentation method were compared with the best results obtained by means of the Masek's segmentation algorithm. Robustness of the method put forward was tested against two iris databases: Casia-irisv3-interval (CASIA3.0) [19] and Ksip-DB01R (KSIP) [20]. The CASIA3.0 contains 2655 eye images sized $320 \times 280$ of 395 irises. The KSIP contains 1920 images sized $352 \times 288$ of 240 irises.

\subsection{Visual Inspection of Localized Iris Boundaries}

The first experiment served to assess the accuracy of the
Table 1 Localization accuracy by human inspection.

\begin{tabular}{ccccc}
\hline & \multicolumn{2}{c}{ Masek } & \multicolumn{2}{c}{ Proposed } \\
\cline { 2 - 5 } Database & CASIA3.0 & KSIP & CASIA3.0 & KSIP \\
\hline Inner Fail (\%) & 4 & 40.4 & 1.2 & 2 \\
\hline Outer Fail (\%) & 9.6 & 18.4 & 0.4 & 0 \\
\hline Inner+Outer Fail (\%) & 0 & 11.2 & 0 & 0 \\
\hline Total Fail (\%) & 13.6 & 47.6 & 1.6 & 2 \\
\hline
\end{tabular}

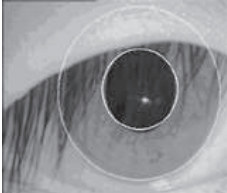

(a)

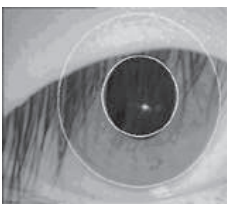

(c)



(b)

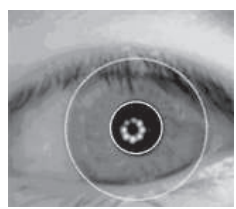

(d)
Fig. 6 Examples of localization results of both databases. The KSIP images are on the left side. The CASIA3.0 images are on the right side. (ab) results obtained with our proposed method, (c-d) results obtained with Masek's method.

proposed localization method. Only inner and outer iris boundaries were visually inspected. For each database, 250 iris images are randomly selected for the inspection. In the experiment arrangement, incorrect localization typically occurs when the obtained boundary differs in excess of 10 pixels from its actual boundary. This difference was measured by overlaying the obtained boundary over the eye image using Photoshop program. Percentages of incorrect localization of inner, outer as well as both of inner and outer boundaries were computed and are displayed in Table 1. The percentage of Total Fail in the table indicates the total number of images of incorrect localized iris boundaries. The Total Fail was attained at with the below equation:

$$
\begin{aligned}
\% \text { TotalFial }= & \% \text { InnerFail }+\% \text { OuterFail } \\
& -\%(\text { Inner }+ \text { Outer }) \text { Fail }
\end{aligned}
$$

The experiment results revealed that the proposed method outperformed Masek's method in both iris databases. Performance of the Masek's algorithm degrades severely when applied to the KSIP iris database. This is due to the higher eyelash occlusions of eye images of the KSIP database. The occlusions disturbed both the inner and outer boundary detection, as shown in Fig. 6. Figure 6(a) and 6 (b) show the results of correctly localized iris boundaries by the suggested segmentation approach. Figure 6 (c) displays the failures of both inner and outer boundary of the image from the KSIP database by the Masek's segmentation approach. From the obtained experimental results, the proposed iris outer boundary based on the LSNR of an edge map proved to be very robust against both databases applied. 
Table 2 Classification performance.

\begin{tabular}{ccccccccc}
\hline & \multicolumn{4}{c}{ Masek } & \multicolumn{4}{c}{ Proposed } \\
\cline { 2 - 8 } Database & CSR & FPR & FNR & AER & CSR & FPR & FNR & AER \\
\hline CASIA3.0 & 94.48 & 3.71 & 10.43 & 7.07 & 95.03 & 5.01 & 4.83 & 4.93 \\
\hline KSIP (\%) & 92.3 & 4.91 & 15.88 & 10.39 & 94.35 & 5.76 & 5.34 & 5.55 \\
\hline
\end{tabular}

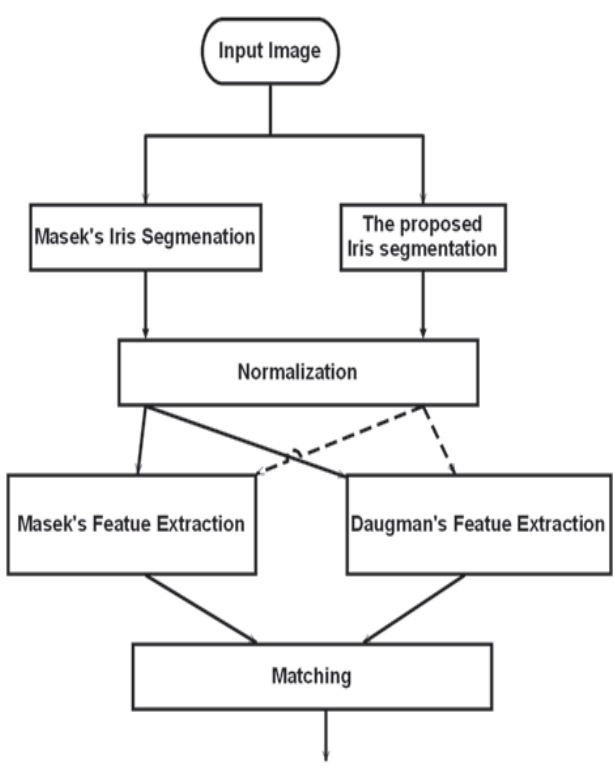

Fig. 7 A protocol of system evaluation.

\subsection{Segmentation Performance via Classification}

The second experiment served to compare the segmented iris with its ground truth image. Two hundred fifty ground truth images of each database were manually generated by categorization of each pixel of the ground truth images as either an iris or non-iris pixel. Then, following classification accuracy were computed: correct segmentation rate (CSR), false positive rate (FPR), false negative rate (FNR) and an average error rate (AER). The CSR is the ratio of a number of correctly classified pixels to a total number of all pixels. The FPR is the ratio of the number of non-iris pixels incorrectly classified as iris pixels to the total number of non-iris pixels. The FNR is the ratio of the number of iris pixels incorrectly classified as non-iris pixels to the total number of iris pixels. The AER is the average value of the FPR and FNR. The obtained results are shown in Table 2 . The results indicated comparable performance in terms of the CSR values. The obtained FRR of the Masek's method is slightly lower than the FRR of our approach. However, the FNR of our approach is approximately half of the Masek's approach.

\subsection{Recognition Performance}

The last experiment is to investigate effects of the proposed iris segmentation on the iris system accuracy. A protocol of this experiment is illustrated in Fig. 7. Two iris segmentation approaches, which are Masek and the proposed segmentation approaches are used with both iris databases for
Table 3 The equal error rate of the proposed system.

\begin{tabular}{ccccc}
\hline & \multicolumn{2}{c}{ Masek's segmentation } & \multicolumn{2}{c}{ Proposed segmentation } \\
\cline { 2 - 5 } Feature Extraction & CASIA3.0 & KSIP & CASIA3.0 & KSIP \\
\hline Daugman (Gabor) & 11.99 & 29.7 & 1.39 & 10.46 \\
\hline Masek (Log Gabor) & 3.1 & 10.08 & 0.85 & 0.46 \\
\hline
\end{tabular}

system performance comparisons. The segmented iris ring is then normalized using Rubber-sheet model [1]. To validate robustness of the segmentation against the feature extraction methods, two feature extraction approaches which are developed by Daugman and Masek are used in the experiment. The normalized iris image is fed to each feature extraction module for the comparison purpose.

Brief details of Daugman's and Masek's feature extraction are as follows. Both Daugmant and Masek represent an iris by extracting phase information of an iris. The obtained phase information is then quantized and encoded using 2 bits. Similarity of two encoded iris codes is evaluated using the Hamming distance. To extract the phase information of an iris, Daugman exploited a 2D Gabor filter, whereas Masek exploited a 1D Log-Gabor filter. An advantage of the Log-Gabor over the 2D Gabor is that the Log-Gabor can be constructed at any arbitrary bandwidth, whereas bandwidth of Gabor is limited to once octave. Therefore, the Log-Gabor has no DC-component. In addition, the Log-Gabor filter contains more high frequency components, resulting in better natural texture analysis [22]. In the experiment, Masek' segmentation and feature extraction approaches are downloaded from [13]. Since there is no public access for Daugman approach [1], the Daugman's feature extraction approach is implemented. The best obtained result by tuning required parameters such as Gabor's frequency is set as the system accuracy.

The iris recognition system accuracy is measured in terms of an equal error rate (EER) with a leave-one-out scheme. The EER is an equilibrium error of Fault Accept Rate (FAR) and Fault Reject Rate (FRR). The comparison results are shown in Table 3 . The results attained prove it evident that the proposed segmentation significantly improves the system performance regardless of the characteristics of an iris database. The experiment confirmed the correlation of the iris system accuracy with the segmentation achievement. However, with the same segmentation approach used, the recognition system performance highly depends on the chosen feature extraction. The results of the performed experiments indicate that Log-Gabor based feature extraction outperforms the Gobor based feature extraction scheme. Although encouraging results were achieved in all three experiments, the false positive rate of the suggested approach should be further reduced. The inaccurate segmentation is due to the non-circular geometry of an iris. Additionally, refinement for better eyelashes removal should be implemented in any future work.

\section{Conclusions}

Details of an iris segmentation based on local image proper- 
ties are described in this paper. The pupil boundary is adaptively segmented with the minimum value of local average values of an eye image. A local property usually referred to as a local signal-to-noise ratio obtained from an edge map of the eye image is introduced for the sclera boundary detection. The LSNR significantly enhances the edges of the sclera boundary, resultant in a robustly detection of the outer iris boundary. The obtained iris boundaries are modeled with two non-concentric circles. Eyelashes and eyelids are removed by adaptive thresholding. The results of assessment against two iris databases without parameter tunings reveal that the proposed segmentation algorithm improves iris recognition system performance significantly.

\section{Acknowledgments}

This work was partially supported by The Thailand Research Fund (TRF) and The Commission on Higher Education (CHE), Ministry of Education under Grant MRG5080293.

\section{References}

[1] J. Daugman, "High confidence recognition of persons by rapid video analysis of iris texture," European Convention on Security and Detection, vol.408, pp.244-251, 1995.

[2] J.V. Forrester, A.D. Dick, P.G. McMenamin, and W.R. Lee, The eye: Basic sciences in practice, Harcourt Publishers, pp.26-31, 2002.

[3] "Information technology - Biometric data interchange formats," Part 6: Iris image data, ISO/IEC 19794-6:2005, 2005.

[4] J. Daugman, "New methods in iris recognition," IEEE Trans. Syst. Man Cybern. B, Cybern., vol.37, no.5, pp.1167-1175, 2007.

[5] A. Ross and S. Shah, "Segmenting non-ideal irises using geodesic active contours," Proc. IEEE Biometric Symposium, p.16, 2006.

[6] E.M. Arvacheh and H.R. Tizhoosh, "Iris segmentation: Detecting pupil, limbic and eyelids," IEEE International Conference on Image Processing, pp.2453-2456, 2006.

[7] Z. He, T. Tan, Z. Sun, and X. Qiu, "Towards accurate and fast iris segmentation for iris biometrics," IEEE Trans. Pattern Anal. Mach. Intell., vol.31, no.9, pp.1670-1684, 2009.

[8] T.N. Tan, Z.F. He, and Z.A. Sun, "Efficient and robust segmentation of noisy iris images for non-cooperative iris recognition," Image and Vision Computing, vol.28, no.2, pp.223-230, Feb. 2010.

[9] W. Sankowski, K. Grabowskia, M. Napieralskaa, M. Zuberta, and A. Napieralskia, "Reliable algorithm for iris segmentation in eye image," Image Vis. Comput., vol.28, no.2, pp.261-269, Feb. 2010.

[10] R. Wildes, "Iris recognition: An emerging biometric technology," Proc. IEEE, vol.85, no.9, pp.1348-1363, 1997.

[11] X.M. Lui, K.W. Bowyer, and P.J. Flynn, "Experiments with an improved iris segmentation algorithm," Proc. 4th IEEE Workshop Automatic Identification Advanced Technologies (AutoID), pp.118123, 2005.

[12] H. Proenca and L.A. Alexandre, "Iris segmentation methodology for non-cooperative recognition," IEE Proc. Vision, Image and Signal Processing, vol.153, no.2, pp.199-205, 2006.

[13] L. Masek and P. Kovesi, "MATLAB source code for a biometric identification system based on iris patterns," The School of Computer Science and Software Engineering, The University of Western Australia, 2003.

[14] W.K. Kong and D. Zhang, "Accurate iris segmentation method based on novel reflection and eyelash detection model," Proc. Int. Sym. Intelligent Multimedia, Video and Speech Processing, Hong Kong, pp.263-266, 2001.
[15] J. Huang, Y. Wang, T. Tan, and J. Cui, "A new iris segmentation method for recognition," Proc. 17th International Conference on Pattern Recognition (ICPR04), Cambridge, pp.554-557, 2004.

[16] R.Y.F. Ng, Y.H. Tay, and K.M. Mok, "An effective segmentation method for iris recognition system," 5 th International Conference on Visual Information Engineering (VIE 2008), pp.548-553, July-Aug. 2008.

[17] N. Sudha, N.B. Puhan, H. Xia, and X. Jiang, "Iris recognition on edge maps," IET Computer Vision, vol.3, no.1, pp.1-7, 2009.

[18] P. Li, X. Liu, L. Xiao, and Q. Song, "Robust and accurate iris segmentation in very noisy iris images," Image and Vision Computing, vol.28, no.2, pp.246-253, Feb. 2010.

[19] http://www.cbsr.ia.ac.cn/english/IrisDatabase.asp accessed Nov. 2009.

[20] http://ksip.ee.ku.ac.th/ accessed Nov. 2009.

[21] C. Sreecholpech and S. Thainimit, "Circular and elliptical modeling for pupil boundary in closed-up human eye images," Proc. Electrical Engineering/Electronics, Computer, Telecommunications, and Information Technology (ECTI) International Conference, pp.445448, 2008.

[22] D.J. Field, "Relations between the statistics of natural images and the response properties of cortical cells," J. Optical Society of America A, Optics and Image Science, vol.4, no.12, pp.2379-2394, 1987.

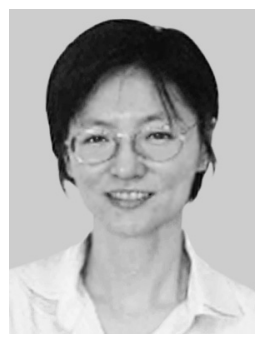

Somying Thainimit received the M.S. degree in Electrical Engineering from Vanderbilt University in USA, in 1997. She received the Ph.D. degree in Computer Engineering from University of Louisiana at Lafayette in USA, in 2002. She is now an assistant professor in Electrical Engineering Depart, Faculty of Engineering, Kasetsart University, Bangkok, Thailand. Her research interests include Image and video processing, face processing, biometrics, computer vision, and computer graphics.

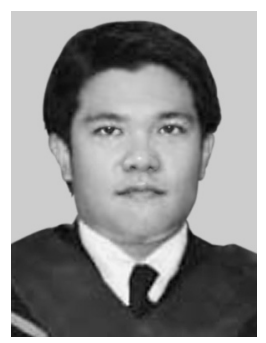

Chirayuth Sreecholpech received the B.S. and MS degree in Electrical Engineering from Kasetsart University in 2005 and 2009, respectively. His research interests include image and video processing and computer vision. 


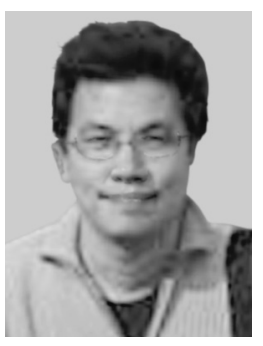

Vuttipong Areekul received the B.Eng. degree in Electrical Engineering from King Mongkut's Institute of Technology, North Bangkok, Thailand in 1990. He received the M.Eng degree in Electrical Engineering from Kasetsart University, Bangkok, Thailand in 1993. He received the Ph.D. degree in Electrical and Computer Engineering from Washington State University, Pullman, USA. in 1998. He is now an associate professor in the electrical engineering department at Kasetsart University. His research interests include fingerprint recognition, biometrics, and agricultural image processing.

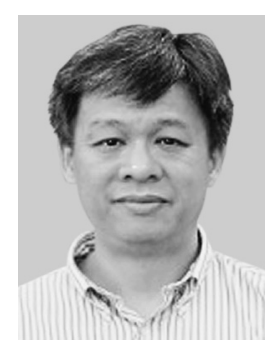

Chee-Hung Henry Chu received the B.S.E. in Computer Engineering and M.S.E. in Computer, Information and Control Engineering from University of Michigan, Ann Arbor in 1981 and 1982, respectively. He received Ph.D. degree in Electrical Engineering, Purdue Univ. in 1988. He is now a professor in The Center for Advanced Computer Studies (CACS), University of Louisiana at Lafayette, USA. His research interests are in pattern recognition, image processing, and machine vision. 